\title{
Erratum to: A Randomized Controlled Study of Parent-assisted Children's Friendship Training with Children having Autism Spectrum Disorders
}

\author{
Fred Frankel · Robert Myatt · Catherine Sugar • \\ Cynthia Whitham • Clarissa M. Gorospe • \\ Elizabeth Laugeson
}

Published online: 20 April 2010

(C) Springer Science+Business Media, LLC 2010

\section{Erratum to: J Autism Dev Disord \\ DOI 10.1007/s10803-009-0932-z}

In the original article, the last two column titles were reversed. The next to last column should be titled $p$ (T2T3), indicating the probability of the change during the follow-up period. The last column should be $p$ (T1-T3) indicating the probability of the change from baseline to the end of the follow-up period (Table 3).
Table 3 Comparison of intervention to follow-up phase for all subjects in the CFT group

\begin{tabular}{llllll}
\hline Variables & $\mathrm{T} 1$ & $\mathrm{~T} 2$ & $\mathrm{~T} 3$ & $p(\mathrm{~T} 2-\mathrm{T} 3)$ & $p(\mathrm{~T} 1-\mathrm{T} 3)$ \\
& $M(\mathrm{SD})$ & $M(\mathrm{SD})$ & $M(\mathrm{SD})$ & & \\
& $n=26$ & $n=26$ & $n=26$ & & \\
\hline Child measures & & & & & \\
Loneliness & $36.3(12.2)$ & $31.6(8.1)$ & $33.0(13.7)$ & $\mathrm{ns}$ & $\mathrm{ns}$ \\
Popularity & $6.9(3.0)$ & $7.9(2.7)$ & $7.4(2.8)$ & $\mathrm{ns}$ & $\mathrm{ns}$ \\
Parent measures & & & & & \\
QPQ & & & & & \\
Host & $2.0(2.1)$ & $4.0(1.6)$ & $3.1(2.9)$ & $\mathrm{ns}$ & $<.05$ \\
Guest & $1.4(1.7)$ & $1.8(2.6)$ & $1.9(1.5)$ & $\mathrm{ns}$ & $\mathrm{ns}$ \\
Conflict & $4.3(3.3)$ & $1.8(3.0)$ & $2.0(2.6)$ & $\mathrm{ns}$ & $<.0005$ \\
Engage & $3.8(2.1)$ & $4.5(2.2)$ & $4.2(2.0)$ & $\mathrm{ns}$ & $\mathrm{ns}$ \\
Disengage & $5.2(2.3)$ & $2.1(1.6)$ & $4.1(2.2)$ & $<.0001$ & $<.025$ \\
SSRS & & & & & \\
Assertion & $9.7(2.8)$ & $11.7(2.8)$ & $12.0(3.5)$ & $\mathrm{ns}$ & $<.0001$ \\
Self control & $9.8(3.5)$ & $12.0(2.8)$ & $11.8(3.8)$ & $\mathrm{ns}$ & $<.005$ \\
Externalizing & $4.5(2.4)$ & $3.8(2.0)$ & $3.8(2.5)$ & $\mathrm{ns}$ & $\mathrm{ns}$ \\
Internalizing & $7.1(1.6)$ & $6.2(1.7)$ & $6.0(2.5)$ & $\mathrm{ns}$ & $<.025$ \\
Teacher measures & & & & & \\
PEI & & & & & $\mathrm{ns}$ \\
Withdrawal & $4.2(2.2)$ & $3.5(2.5)$ & $3.6(2.8)$ & $\mathrm{ns}$ & $\mathrm{ns}$ \\
Aggression & $1.5(1.8)$ & $1.3(1.3)$ & $1.6(1.7)$ & $\mathrm{ns}$ & $\mathrm{ns}$ \\
\hline
\end{tabular}

a $n=24$

The online version of the original article can be found under doi:10.1007/s10803-009-0932-z.

F. Frankel $(\bowtie) \cdot$ R. Myatt - C. Sugar - C. Whitham .

C. M. Gorospe · E. Laugeson

UCLA Semel Institute For Neuroscience and Human Behavior,

300 UCLA Medical Plaza, Los Angeles, CA 90095, USA

e-mail: ffrankel@mednet.ucla.edu

C. Sugar

Department of Biostatistics, UCLA School of Public Health,

Center For Health Sciences, Los Angeles, CA 90095-1772, USA 